\title{
Job Shop Scheduling Focusing on Role of Buffer*
}

\author{
Rei HINO**, Tetsuya KUSUMI***, Jae-Kyu YOO ${ }^{* * *}$ and Yoshiaki SHIMIZU***
}

\begin{abstract}
A scheduling problem is formulated in order to consistently manage each manufacturing resource, including machine tools, assembly robots, AGV, storehouses, material shelves, and so on. The manufacturing resources are classified into three types: producer, location, and mover. This paper focuses especially on the role of the buffer, and the differences among these types are analyzed. A unified scheduling formulation is derived from the analytical results based on the resource's roles. Scheduling procedures based on dispatching rules are also proposed in order to numerically evaluate job shop-type production having finite buffer capacity. The influences of the capacity of bottle-necked production devices and the buffer on productivity are discussed.
\end{abstract}

Key Words: Buffer Capacity, Scheduling, WIP (Work In Process), Bottle Neck

\section{Introduction}

Semi-finished products must be temporarily stored due to differences of processing time and order in multistep manufacturing processes. A shelf is a typical of manufacturing resource for storing semi-finished products, but an area marked by painted lines is often used for this purpose in certain factories. In this paper, a buffer is defined as a manufacturing resource for reserving material, semifinished products, and products, and a job-shop scheduling problem involving a buffer having finite capacities is studied.

Previous investigations have focused on scheduling problems involving finite buffer capacities. For example, a scheduling problem with a dedicated buffer for each product has been examined by Tamaki et al. ${ }^{(1),(2)} \mathrm{Fu}$ et al. also studied a case involving a commonly used buffer ${ }^{(3),(4)}$. However, these conditions are special from an actual manufacturing point of view.

In the present paper, a new type of scheduling problem is formulated based on the buffers used in general manufacturing activities. The formulation is derived from the roles of three types of manufacturing resources, namely, production devices, conveyance devices,

* Received 22nd March, 2006 (No. 04-0571). Japanese Original: Trans. Jpn. Soc. Mech. Eng., Vol.71, No.702, C(2005), pp.685-692 (Received 18th May, 2004)

** Department of Mechanical Science and Engineering, Nagoya University, Furo-cho, Chikusa-ku, Nagoya, 4648603, Japan. E-mail: hino@mech.nagoya-u.ac.jp

*** Department of Production Systems Engineering, Toyohashi University of Technology, 1-1 Hibarigaoka, Tenpaku-cho, Toyohashi 441-8580, Japan and buffers. A new dispatching procedure is also proposed in order to create an appropriate schedule in light of this new scheduling problem.

\section{Scheduling Focusing on Storage Time}

\subsection{Nomenclature}

In this paper, a job (which is regarded as a primitive unit in the scheduling problem) is called a "process" in order to emphasize the content for the product's manufacture. It is also called an "operation" in order to emphasize the content handled by the machine. The word "job" is used for the buffer's storing of materials, semi-finished products, and products.

The following symbols are used for representations of the information processing method proposed here. Subscripts are used for representing information regarding both product and production resource, but certain subscripts are omitted if no information gives. Further details of the representation nomenclature will be described as the need arises.

$j_{\eta, i}^{\zeta, v}: v$ th operation processed by machine $\zeta$

and $i$ th process for product $\eta$.

$\bar{j}_{\eta, i}^{\zeta, v}:$ reservation of $j_{\eta, i}^{\zeta, v}$

$s, f, p:$ start, finish and duration time for processing of $j$

$\bar{s}, \bar{f}, \bar{p}:$ start, finish and duration time for reservation of $j$

P. Gullander et al. categorized manufacturing resources into the following three types to create a model of the manufacturing system ${ }^{(5)}$.

A Producer makes necessary physical or logical changes to product properties. 


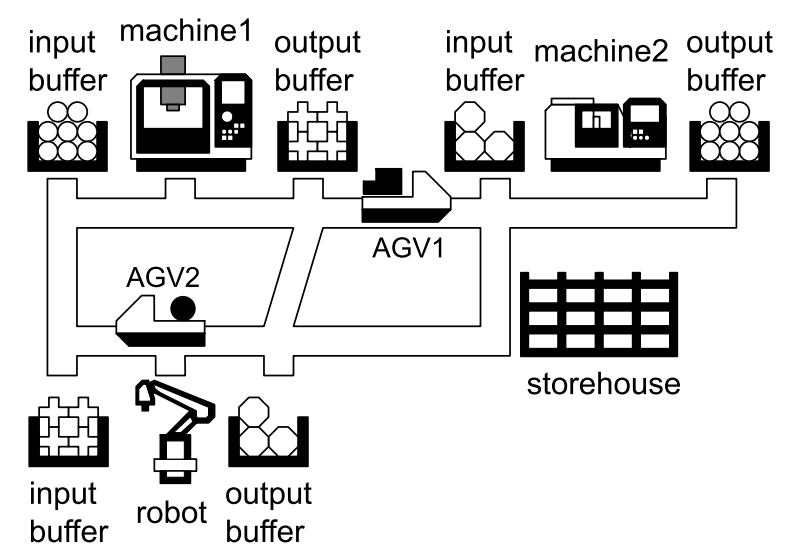

Fig. 1 Production system including buffer

A Location only reserves products and cannot change any properties of the products.

A Mover transports the products between producers and locators.

In this study, the scheduling problem is analyzed in terms of these three categories, and we try to clarify the role of individual manufacturing resources in a manufacturing. According to P. Gullander's categories, a "producer" includes production devices such as machine tools and robots, and "location" means a buffer, which is the subject of this paper. "Mover" includes automated guided vehicles (AGVs) and conveyer belts. A container is defined as a unit resource that can reserve just one job. One buffer consists of multiple containers; therefore, the capacity of the buffer equals the number of containers. The "manufacturing resource" is the general term for these devices. Put another way, every manufacturing resource can be categorized into one of three different types.

\subsection{Basic structure of the production system}

Figure 1 shows a schematic illustration of a production system in which every production device has both an input buffer and an output buffer which reserve row materials, semi-finished materials, and products. The production devices take the materials from the buffer and also receives them directly from conveyance devices. In the same way, they place the semi-finished products onto a buffer and also leave them on conveyance devices. In addition to the exchange of the semi-finished product between production devices, the conveyance device, and the buffer, we take account the exchange between production devices and conveyance devices in order to formulate the scheduling problem without any buffers. Fu et al. focuses on a common buffer illustrated in Fig. 1 as a storehouse in a production system without conveyance devices ${ }^{(3),(4)}$.

\subsection{Influence of preceding operation}

In a conventional scheduling problem, in addition to the condition that one manufacturing resource can only handle one job at a time, the following two presumptions are important ${ }^{(6)}$. a) resource sequences for production are given.

b) processing time for each process is given.

Under these two presumptions, a scheduling problem is defined for a manufacturing system composed of only production devices. When conveyance devices are adopted in addition to the production devices, the scheduling procedure must be modified in order to take account of the influence of the preceding operation. When the resource sequences including conveyance (condition a) and the transportation time (condition $b$ ) are given, the schedule can be planned as a conventional procedure. However, the conveyance device must move to receive toward the resource that finishes the process. The moving time is the same duration as the transportation time, and in order to create a correct schedule the moving time cannot be disregarded. The moving time depends on the position of the conveyance device, or it is influenced by the preceding operation of the conveyance device. Therefore, the moving time is determined during the scheduling procedure ${ }^{(7)}$.

After the introduction of the modification, a time corresponding to the moving time of the conveyance device is identified regarding the production device. In a conventional scheduling problem, in general the time might be ignored due to its brevity, or it can be included in processing time because its value is constant.

In contrast, the influence of the processing order of the production device cannot be disregarded; the same modified procedure must be adopted to create the schedule in a system composed of only production devices. A painting process is a typical example of the influence of the preceding operation. The painting machine can immediately begin a new operation if it is to paint the same color as the preceding one; however, it must wash its nozzle if it is to apply a different color.

\subsection{Influence of succeeding process}

The role of the buffer (location) is to temporarily reserve row materials, semi-finished products during production, and finished products after production. The reservation time is defined as the duration from the time the semi-finished product processed is received by the production device to [the time it leaves] with another production device assigned to the succeeding process. Obviously, the reservation time cannot be determined before the schedule is planned.

Figure 2 (a) shows an illustration of a manufacturing system composed of three essential production resources. A container $\psi$ reserves the semi-finished product after a production device machine $\xi$ processes it, and a conveyance device $\mathrm{AGV} \zeta$ then receives it for transportation. As shown in the Fig. 2 (b), the reservation of the job $\bar{j}_{\beta, j}^{\psi, w}$ by container $\psi$ begins immediately after finishing reserving the preceding operation and the container must maintain its status until leaving the reserved product with AGV $\zeta$. Limited buffer capacity causes the same constraint to 


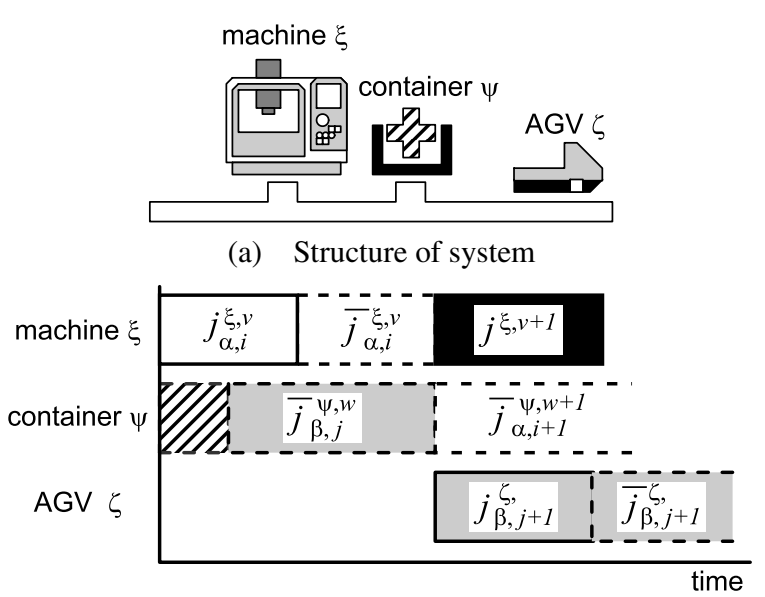

(b) Example of schedule

Fig. 2 Gantt chart focusing on reservation of job

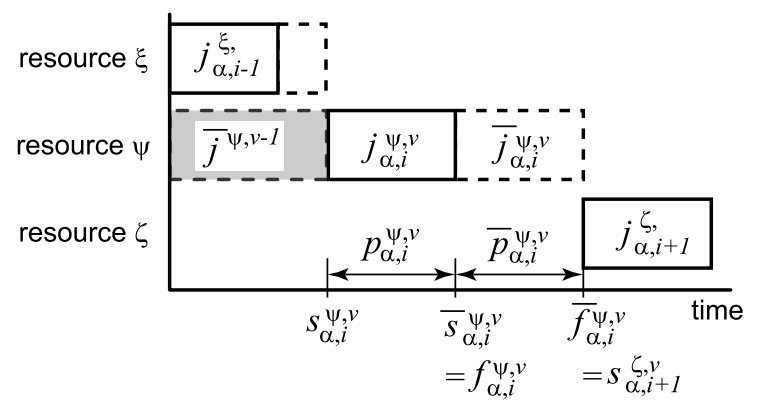

Fig. 3 Scheduling time decided among three resources

the production device. For example, the machine $\xi$ must wait to operate the job $j^{\xi, v+1}$ until the container $\psi$ begins to reserve of the job $\bar{j}_{\alpha, i+1}^{\psi, w+1}$, which is represented as the successor process of the job $j_{\alpha, i}^{\xi, v}$. Obviously, the job $j_{\alpha, i}^{\xi, v}$ remains on the machine $\xi$ until the starting time of the reservation of $\bar{j}_{\alpha, i+1}^{\psi, w+1}$. The status of the $\bar{j}_{\alpha, i}^{\xi, v}$ corresponds to the reservation by the container $\psi$. This situation has to do with the conveyance device.

In the previous section, we discussed the roles of the production device and conveyance device in the scheduling procedure. For the reasons mentioned above, the buffer is also identified as a manufacturing resource. That is to say, the reservation status must be taken into account when regarding the production device and conveyance device. Return to the discussion about the buffer, the buffer can be handled as the manufacturing resource processing nothing, or the processing time is zero in scheduling procedure.

\subsection{Formulation of preparation and reservation}

Stating time, reserve time, and finishing time are formulated according to the influence of both preceding operations and succeeding processes. The formulation here is based on the relationship between the three manufacturing resources shown in Fig. 3.

First of all, a later time is calculated from the finishing time of the preceding job $j^{\psi, v-1}$ operated by resource $\psi$ and the preceding job $j_{\alpha, i-1}^{\xi}$ processed by another resource $\xi$. The stating time of the job $j_{\alpha, i}^{\psi, v}$ is determined by using a time defined by both the job $j_{\alpha, i}^{\psi, v}$ and the preceding job $j^{\psi, v-1}$.

$$
s_{\alpha, i}^{\psi, v}=\max \left(\bar{f}^{\psi, v-1}, f_{\alpha, i-1}^{\xi,}\right)+F\left(j^{\psi, v-1}, j_{\alpha, i}^{\psi, v}\right),
$$

where $\max ()$ indicates the function for calculating the later time within brackets. The function $F()$ is defined by each manufacturing resource. For an example, the following function $F()$ can be defined concerning the conveyance device $^{(7)}$,

$$
F\left(j^{\psi, v-1}, j_{\alpha, i}^{\psi, v}\right)=l_{v, \mu} / v_{\psi},
$$

where, the job $j^{\psi, v-1}$ represents one conveyance from a resource $v$ to another resource. The job $j_{\alpha, i}^{\psi, v}$ also represents a conveyance for a resource $\mu$ to another resource. The symbol $l_{v, \mu}$ indicates the distance from the resource $v$ and $\mu$, and $v_{\psi}$ is the velocity of the conveyance device $\psi$.

The job's finishing time is defined by the following equation using the value given by presumption.

$$
f_{\alpha, i}^{\psi, v}=s_{\alpha, i}^{\psi, v}+p_{\alpha, i}^{\psi, v} .
$$

The starting time of the reservation equals the finishing time of the job.

$$
\bar{s}_{\alpha, i}^{\psi, v}=f_{\alpha, i}^{\psi, v} .
$$

The finishing time of the reservation equals the starting time of the successor process $j_{\alpha, i+1}^{\zeta}$ assigned to the resource $\zeta$

$$
\bar{f}_{\alpha, i}^{\psi, v}=s_{\alpha, i+1}^{\zeta,} .
$$

The reserving time is given as follows:

$$
\bar{p}_{\alpha, i}^{\psi, v}=\bar{f}_{\alpha, i}^{\psi, v}-\bar{s}_{\alpha, i}^{\psi, v} .
$$

\subsection{Unfeasible schedule}

There exists some unfeasible schedules because the starting time of the jobs cannot be determined due to the inadequate processing order of the operations. Fu discusses deadlock status under the condition without a buffer and that with limited buffer capacities ${ }^{(3),(4)}$. In this paper, we argue reservation by the production device and conveyance device in addition to the buffer; therefore, other unfeasible schedules are discussed.

Figure 4(a) shows an unfeasible schedule for two products processed by two manufacturing resources in the same sequences. Under this schedule, a single resource cannot change the processing order. For example, resource $\psi$ cannot process the job $j_{\beta, j+1}^{\psi,}$ at $w$ th, and the job $j_{\alpha, i+1}^{\psi,}$ at $w+1$ th as shown in Fig. 4(a). The resource $\xi$ can begin to process the job $j_{\beta, j}^{\xi, v+1}$ after the resource $\psi$ receives the preceding job $j_{\alpha, i}^{\xi, v}$ processed by the resource $\xi$. Therefore, the resource $\psi$ cannot process the job $j_{\beta, j+1}^{\psi,}$ before the job $j_{\alpha, i+1}^{\psi,}$. This constraint is subjected to not only the buffer but also to the production device and the conveyance device. 


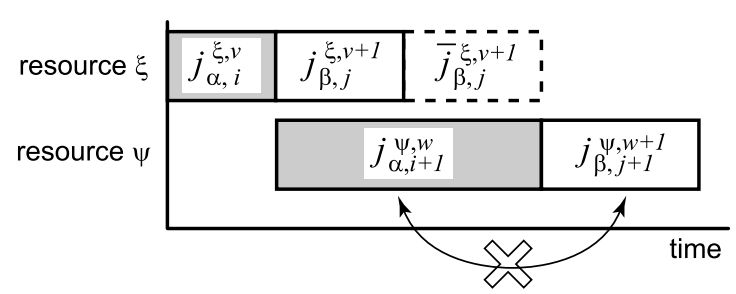

(a) Prohibition of exchange of operating order

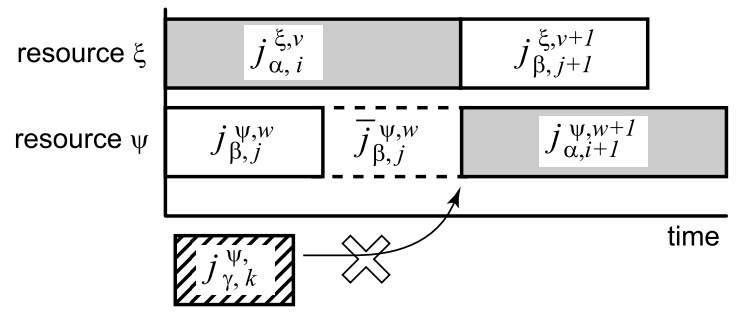

(b) Prohibition of insertion of another job

Fig. 4 Examples of procedure that causes unfeasible schedule

Figure 4(b) shows another unfeasible schedule in which two resources exchange jobs with each other. For an example, a conveyance device transfers a new task to a production device and receives a finished task processed by the production device simultaneously. Although Fu prohibits this schedule in his scheduling procedure, we accept this exchange between two resources. However, another new job $j_{\gamma, k}^{\psi,}$ cannot be inserted between the job $j_{\beta, j}^{\psi, w}$ and $j_{\alpha, i+1}^{\psi, w+1}$. This is for the same reason as the case shown in Fig. 4 (a).

\section{Dispatching Rule for Schedule with Reservation}

In order to confirm the feasibility of the planned schedule involving reservation by the manufacturing resources, we propose a new criterion for dispatching jobs to the respective resources.

As a basic rule, no new job can be assigned immediately after the job whose duration of reservation is not determined. However, one job can be temporarily assigned to a resource when no resource can accept a new job because the time of the job assigned last is not determined, although only one job is given a temporary assignment. As an exception to this rule, when two jobs are exchanged between two resources, these jobs are assignable even if there reservation times are not determined. The proposed scheduling procedure consists of two routines, or choosing jobs assuring the feasibility of the schedule and dispatching one job to one resource.

\subsection{Choice of jobs assuring schedule feasibility}

initialization Three sets $\boldsymbol{A}, \boldsymbol{B}$ and $\boldsymbol{C}$ are defined. Set $\boldsymbol{A}$ includes either the first process or a job whose previous process is assigned to a resource for every product. Set $\boldsymbol{B}$ consists of jobs assuring the feasibility of the schedule after assigning the job to the resource; at the initial step, $\boldsymbol{B}=\phi$. Set $\boldsymbol{C}$, in contrast, consists of the job causing the unfeasible schedule by assigning the job to the resource; initially $\boldsymbol{C}=\phi$.

Step 1 Choose a job $j_{\alpha, i}^{\xi, *}$ form set $\boldsymbol{A}$, where $*$ indicates the order of the job is undecided regarding the resource $\xi$. A $j_{\beta, j}^{\xi, v}$ is the last job assigned to the resource $\xi$ at this point. If the time $\bar{f}_{\beta, j}^{\xi, v}$, which is the conclusion of the reservation of the job $j_{\beta, j}^{\xi, v}$, is determined, the job $j_{\alpha, i}^{\xi, *}$ is moved from set $\boldsymbol{A}$ to $\boldsymbol{B}$. This operation is accomplished regarding all the jobs included in set $\boldsymbol{A}$.

Step 2 According to the next routine described as a scheduling procedure in Step 8, one of the jobs in set $\boldsymbol{B}$ is assigned to a resource, if there exists at least one job. On the other hand, no assignable job might be found because none of the finishing times of the reservation of any of last jobs assigned to the resource have yet been determined. A job assuring the feasibility of the schedule is extracted by the procedure described as Step 3. That is to say, if the set $\boldsymbol{B}$ is not empty, then the first half of the procedure is completed, otherwise move the procedure described in Step 3.

Step 3 Extract the job $j_{\alpha, i}^{\xi, *}$ from set $\boldsymbol{A}$, record the job as $\breve{j}$, and move to Step 4 .

Step 4 If the job $j_{\beta, j}^{\xi, v}$ processed by the resource $\xi$ is included in set $\boldsymbol{B}$, the schedule is unfeasible, or if $j_{\beta, j}^{\xi, v} \in \boldsymbol{B}$ then move to Step 5, otherwise move to Step 6.

Step 5 Return both set $\boldsymbol{A}$ and $\boldsymbol{B}$ to the status after the procedure is completed at Step 1. The job $\breve{j}$ is taken from set $\boldsymbol{A}$ and placed into $\boldsymbol{C}$. Return to Step 3 .

Step 6 Add the job $j_{\alpha, i}^{\xi, *}$ into set $\boldsymbol{B}$ as $v+1$ th job operated by resource $\xi$. If the next process of job $j_{\beta, j}^{\xi, v}$, which means $v$ th job operated by the resource $\xi$, is included in set $\boldsymbol{B}$, the schedule is feasible. Therefore, if $j_{\beta, j+1}^{\psi, w} \notin \boldsymbol{B}$ then move to Step 7, otherwise move to Step 8.

Step 7 Assign job $j_{\alpha, i}^{\xi, *}$ to the resource $\xi$ as the $v+$ 1th operation, and temporarily calculate the starting time $s_{\alpha, i}^{\xi, v+1}$, finishing $f_{\alpha, i}^{\xi, v+1}$, and starting time of the reservation $\bar{s}_{\alpha, i}^{\xi, v+1}$. For this calculation, assume that the finishing time of the reservation $\bar{f}_{\beta, j}^{\xi, v}$ of job $j_{\beta, j}^{\xi, v}$, which means the last job operated by the resource $\xi$ at this point equals the starting time $\bar{s}_{\beta, j}^{\xi, v}$.

If the next process of job $j_{\alpha, i}^{\xi, v+1}$, which is described as $j_{\alpha, i+1}^{\zeta, *}$, exists, add it to set $\boldsymbol{A}$.

Focus on the job $j_{\beta, j}^{\xi, v}$, which is the previous operation of the job $j_{\alpha, i}^{\xi, v+1}$ assigned to the resource $\xi$, and then take the next process $j_{\beta, j+1}^{\varphi, *}$ of the job as job $j_{\alpha, i}^{\xi, *}$, back to the procedure described in Step 4.

The second routine is for the assignment of the job in set $\boldsymbol{B}$, which assures the feasibility of the schedule taking into account the reservation. Any conventional dispatching rules are available for application here.

\subsection{Scheduling procedure}

Step 8 Select only one job $j_{\alpha, i}^{\xi, *}$ from set $\boldsymbol{B}$ according 
Table 1 Job sequence

product 1: resource $1(10) \rightarrow$ resource $3(10)$

product 2: $\quad$ resource $2(15) \rightarrow$ resource $3(20)$

product $3: \quad$ resource $3(20) \rightarrow$ resource $2(10)$

number in () shows processing time

to a dispatching rule.

Step 9 Determine the starting time $s_{\alpha, i}^{\xi, v+1}$, finishing time $f_{\alpha, i}^{\xi, v+1}$, and starting time of the reservation $\bar{s}_{\alpha, i}^{\xi, v+1}$ for the job $j_{\alpha, i}^{\xi, *}$ according to Eqs. (1), (3) and (4). If the job $j_{\alpha, i-1}^{\psi, w}$ which represents the previous process of the job $j_{\alpha, i}^{\xi, v}$, exists, then fix the finishing time of reservation $\bar{f}_{\alpha, i-1}^{\psi, w}$ by Eq. (5).

Step 10 Finish the scheduling procedure if all jobs are assigned to resources, otherwise return to Step 1 and renew set $\boldsymbol{B}$.

An example of the proposed scheduling procedure is explained. Table 1 shows a simple problem involving three products processed by three resources. Every product is produced by two of three resources. Numbers in ( ) shown in the table indicate the processing time occupying the resource.

First processes for every product are selected for set $\boldsymbol{A}$ initially, or $\boldsymbol{A}=\left\{j_{1,1}^{1, *}, j_{2,1}^{2, *}, j_{3,1}^{3, *}\right\}$. At this point, set $\boldsymbol{B}=\boldsymbol{A}$, because no job is assigned to a resource. As an example of the dispatching rule, a job $j_{1,1}^{1, *}$, which can be finished at the earliest time, is selected. Consequently, a job $j_{1,2}^{3, *}$, which is the next process of the job $j_{1,1}^{1, *}$, is added into set $\boldsymbol{A}$, or set $\boldsymbol{A}=\left\{j_{1,2}^{3, *}, j_{2,1}^{2, *}, j_{3,1}^{3, *}\right\}$, when set $\boldsymbol{B}$ is modified.

Figure 5 (a) shows a schedule after three processes are assigned to the resources according to Step 1 and Step 2. The finishing time of the reservation of every process is not determined regarding each resource, and set $\boldsymbol{B}=\phi$. Therefore, set B is formed according to the procedure following Step 3.

First, $j_{1,2}^{3, *}$ is selected as one candidate, and the job is Added to set $\boldsymbol{B}$ as $\breve{j}=j_{1,2}^{3, *}$. The schedule after the assignment of $j_{1,2}^{3, *}$ is shown in Fig. 5 (b). Second, at Step 7 consequent to Step 4 and Step 6, $j_{3,2}^{2, *}$, which is the next process of the job $j_{3,1}^{3,1}$ assigned to resource 3 , is selected and assigned to resource 2 . The job $j_{2,2}^{3, *}$ is also selected in the same way.

At this point, job $j_{1,2}^{3,2}$ is the last operation processed by resource 3 , which processes the job $j_{2,2}^{3, *}$, and the job is included in set $\boldsymbol{B}$. Therefore these jobs lead to an unfeasible schedule according to the determination in Step 4, and

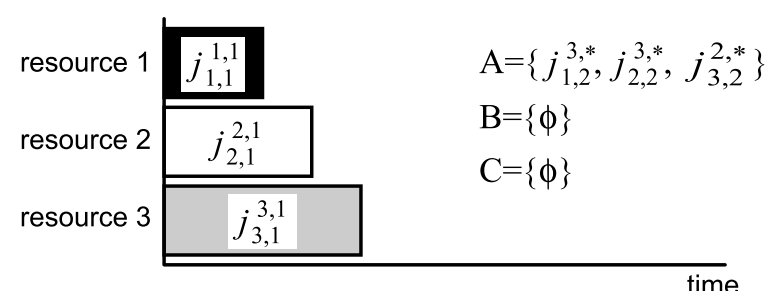

(a) Gantt chart assigned first process of every job

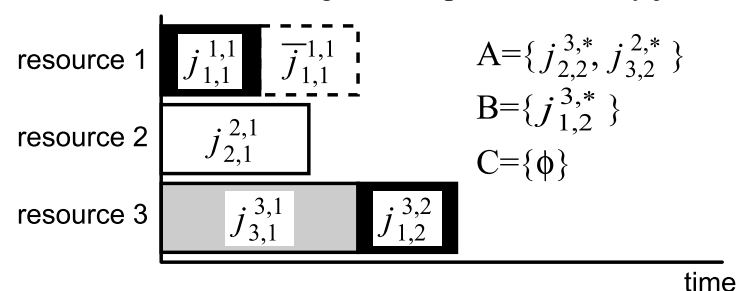

(b) Selection of assignable jobs

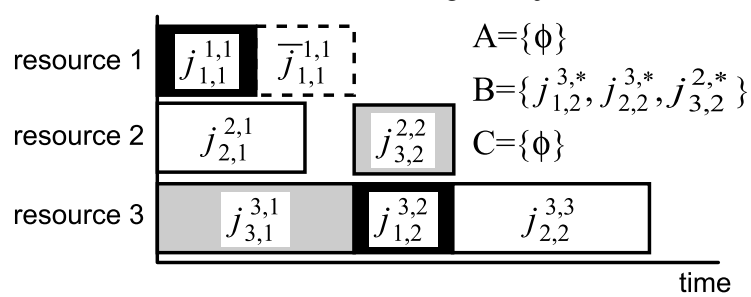

(c) Detection of infeasible combination

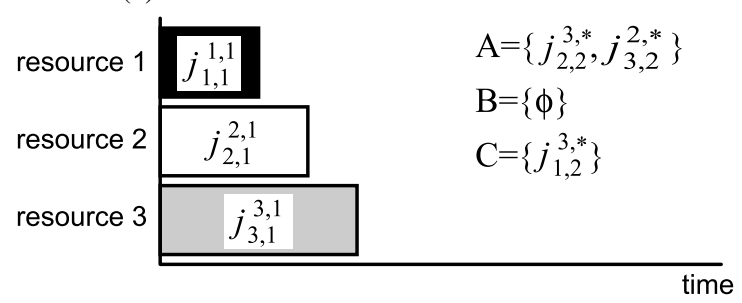

(d) Re-selection of assignable jobs

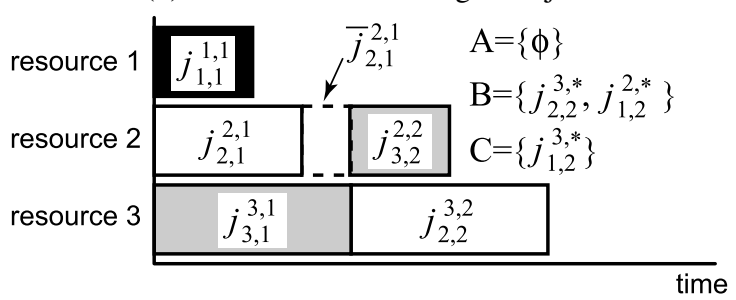

(e) Detection of feaible combination

Fig. 5 Example of procedure according to proposed algorithm

the job $\breve{j}=j_{1,2}^{3, *}$ is moved to set $\boldsymbol{C}$ at Step 5. The schedule and the sets are shown in Fig. 5 (d).

Figure 5 (c) shows an unfeasible schedule after the $\breve{j}=$ $j_{1,2}^{3, *}$ is assigned to resource 3 . The schedule corresponds to the prohibition as shown in Fig. 5 (d), or a job is inserted between two jobs exchanged by two resources each other.

Figure 5 (e) shows the schedule obtained after the job $j_{3,2}^{2, *}$ is selected as the job $\breve{j}$, and assigned to resource 2 . The set $\boldsymbol{B}$ contains the jobs assuring the feasibility schedule.

The job is also assigned to resource 3 and the schedule procedure is completed.

\section{Computational Simulations}

Computational simulations are examined in order to 


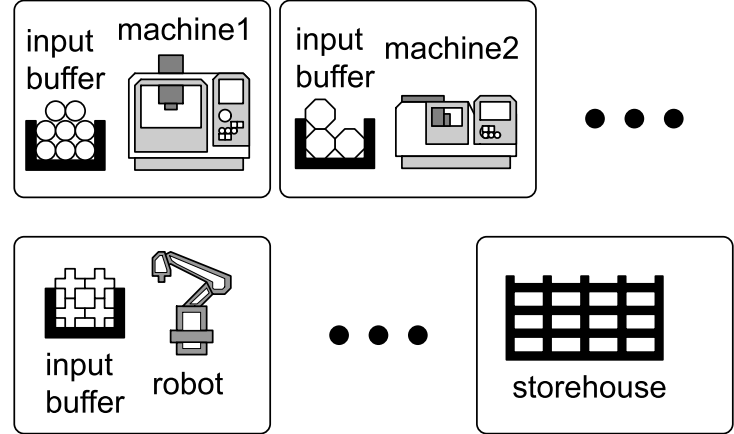

Fig. 6 An example of production system

verify the proposed scheduling problem involving reservation by the manufacturing resources. For simplification of the scheduling problem, the conveyance is not taken into account. Furthermore, in order to avoid continuous reservation from the output buffer of the resource to the input buffers of another resource, the output buffers are not prepared. Figure 6 shows a schematic illustration of the system, and every production device has its own input buffer. Therefore, the semi-finished product processed by the resource is given to another resource directly or reserved in the input buffer of another resource. The buffer consists of multiple containers and each container can reserve one job at a time. Each buffer consists of different numbers of containers, or the capacity of the buffer equals the number of containers. The products which have completed all processes are reserved in a storehouse which is one of buffers, and the capacity of the storehouse is more than the number of products as per the assumption.

In scheduling, either one resource or its input buffer is selected as a manufacturing resource after another resources finishes the operation. In this study, a more profitable resource is selected according to the evaluation to both the resource and its buffer. If the buffer consists of multiple containers, every container is evaluated for selection. A dispatching rule, called the earliest stating time of the job, is adopted for the scheduling in this study.

Job-shop scheduling problems are examined for manufacturing by ten production devices for one hundred products. Every product is processed by every resource at one time and the scheduling problems are generated using numbers generated randomly by a computer. We evaluate the average value of one thousand scheduling results. It is assumed that one production device has low performance in comparison with the others, or that the device takes twice the average processing time.

The capacity of the buffer, or the number of the containers, is changed in scheduling in order to clarify the role of the buffer. At the beginning of the computational simulations, no production device has a buffer as an initial state. Consequently, the reservation time for each job is evaluated in terms of the planned schedule. As discussed

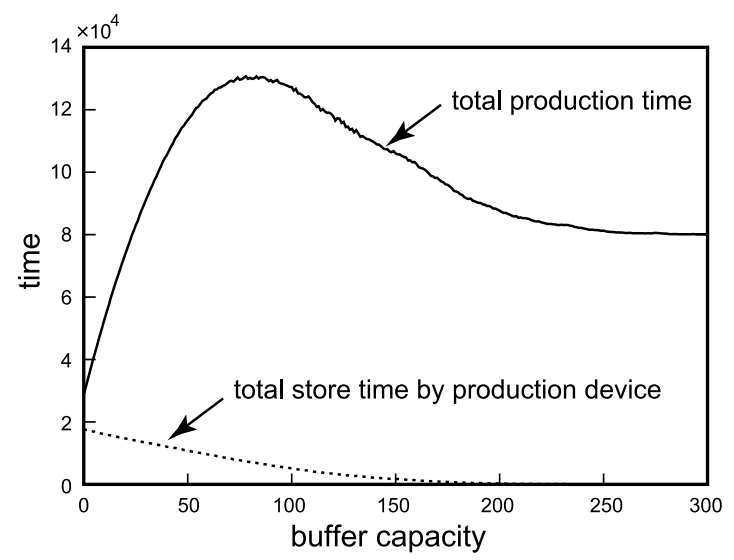

(a) Production time and store time

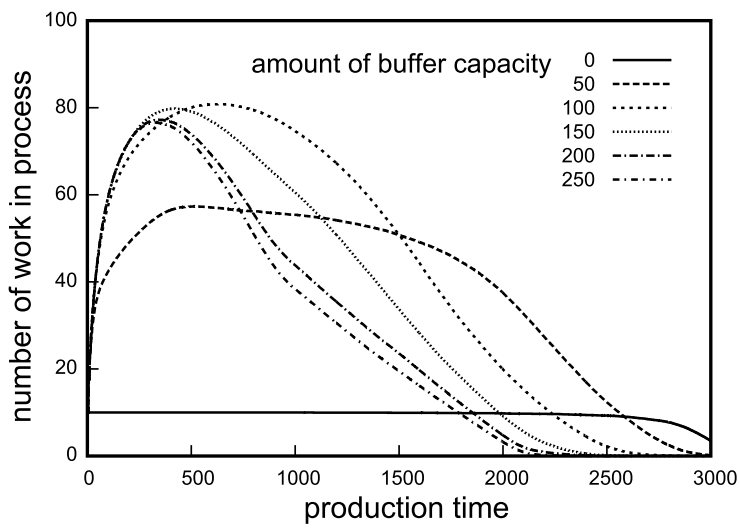

(b) Transition of number of work in process

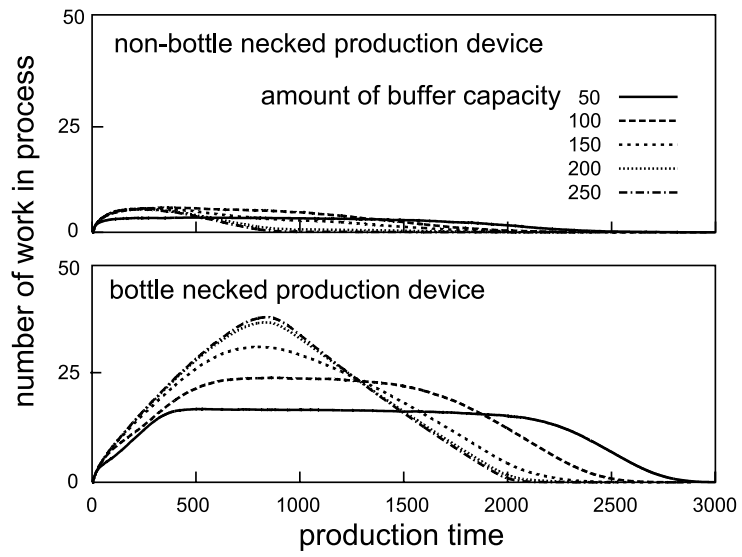

(c) Transition of number of work in process on bottle necked and non-bottle necked production device

Fig. 7 Examples of simulation results

in section 2, the reservation status is determined when another resource cannot receive any other jobs. Therefore, increasing the number of containers comprising the input buffer of the next production device is effective in reducing the job's reservation time.

Figure 7 shows the computational results. As seen in Fig. 7 (a), the reservation times of the jobs in terms of the production devices tends to decrease corresponding to the increment of the buffer capacities. Total production time, i.e., the sum of the time until every finished product 
is delivered to the storehouse, increases once and maintains a constant value after the reservation time become zero. Figure 7 (b) shows the number of the semi-finished products, or WIP (works in process), on every production device and buffer from the beginning of the operation. As the buffer capacity increases, the time necessary for completing the processes of all products decreases. Furthermore, the number of WIP increases according to increment of buffer capacities. A production device can reserve the semi-finished product to begin the operation of a new job because it contains sufficient buffer capacity. Furthermore, it can choose the appropriate job to shorten total production time. This means that the waiting times of some products become longer. Total production time at a maximum buffer capacity of 100 also supports our argument as shown in Fig. 7 (a).

Figure 7 (c) shows the relationship between number of WIP reserved in the buffer during production time and buffer capacity regarding non-bottle-necked and bottlenecked production devices, respectively. A larger number of semi-finished products stays in the buffer of the bottlenecked production devices than in the non-bottle-necked one. However, the number of semi-finished products remains constant in both of them when the buffer capacity is small. On the other hand, corresponding to the increment of buffer capacity, the number of WIP reached a peak immediately after the start of production by the non-bottlenecked production device, while the bottle[-]necked production device reached its peak later. That is to say, even if the production device has high productivity it cannot begin on a new job until the succeeding production device receives its product, because of low buffer capacity.

It is not altogether true that the increment of buffer capacity contributes to the reduction of total production time. The buffer capacity should rather be limited than increased in order to maintain the amount of WIP, because production devices cannot begin a new operation until the succeeding production devices or buffers receive the semifinished products. This corresponding to the style of production in TOYOTA systems in which a new operation proceeds according to the succeeding processes.

In this paper, it is clear that both production devices and buffers can be categorized as the same manufacturing resource from a scheduling point of view. As shown by the results of computational simulations, the amount of buffer capacity is related closely to the reservation time by the production device in the preceding operation. Since the production device is used after the reservation by the buffers, the performance of production devices must be improved in order to reduce the amount of reservation in the buffers. This is also supported by the Theory of Constraints (TOC) ${ }^{(9)}$.

\section{Conclusions}

In this paper, production devices, conveyance devices, and buffers are identified as a same manufacturing resource from a scheduling view point. The results of this paper can be summarized as follows.

1) A new scheduling problem commonly adopted for production devices, conveyance devices, and buffers is formulated focusing on reservation time and preparation time.

2 ) A scheduling procedure is proposed for planning a feasible schedule including buffer capacity.

3 ) The role of a buffer in manufacturing activities is investigated using computational simulations. As proof of the validity of the proposed scheduling technique, it is shown that the production is able to be so executed as to keep amount work in process constant by controlling buffer capacities.

\section{References}

( 1 ) Tamaki, H. and Nishikawa, Y., Modeling of Job-Shop Scheduling Problem with In-Process Buffer Capacity, Transactions of the Society of Instrument and Control Engineers, (in Japanese), Vol.31, No.7 (1995), pp.933940.

( 2 ) Tamaki, H. and Nishikawa, Y., Solution of the JobShop Scheduling Problem with Consideration of InProcess Buffer Capacity, Transactions of the Society of Instrument and Control Engineers, (in Japanese), Vol.31, No.8 (1995), pp.1193-1201.

( 3 ) Fu, G., Zhao, Y. and Sannomiya, N., Application of Genetic Algorithm to No-Buffer Jobshop Scheduling Problems, Transactions of the Institute of Systems, Control and Information Engineers, (in Japanese), Vol.16, No.5 (2003), pp.234-241.

(4) Fu, G., Zhao, Y. and Sannomiya, N., A Method for Solving Jobshop Scheduling Problems with Limited Common Buffers, Transactions of the Institute of Systems, Control and Information Engineers, (in Japanese), Vol.17, No.3 (2004), pp.113-121.

( 5 ) Gullander, P., Fabian, M., Andreasson, S.-A., Lennartson, B. and Adlemo, A., Generic Resource Models and a Message-Passing Structure in an FMS Controller, IEEE International Conference on Robotics and Automation, (1995), pp.1447-1454.

( 6 ) Brucker, R., Scheduling Algorithms, (2001), Springer.

( 7 ) Hino, R. and Moriwaki, T., Resource Reallocation Based on Production Scheduling(1st Report) (Adoption of Schedule as Index for Resource Allocation), Journal of the Japan Society for Precision Engineering, (in Japanese), Vol.69, No.5 (2003), pp.655-659.

( 8 ) Just-In-Time Manufacturing System Workshop, JustIn-Time Manufacturing, (in Japanese), (2004), Nikkan Kogyo Shimbun, LTD.

(9) Goldratt, E.M. and Cox, J., The Goal, A Process of Continuous Improvement, (1994), North River Press. 